\title{
Generation of high-energy neutron beam by fragmentation of relativistic heavy nuclei
}

\author{
Vladimir Yurevich \\ Laboratory of High Energy Physics, Joint Institute for Nuclear Research \\ 6 Joliot Curie, Dubna, Moscow region 141980, Russian Federation \\ yurevich@jinr.ru
}

Published 1 September 2016

\begin{abstract}
The phenomenon of multiple production of neutrons in reactions with heavy nuclei induced by high-energy protons and light nuclei is analyzed using a Moving Source Model. The Lorentz transformation of the obtained neutron distributions is used to study the neutron characteristics in the inverse kinematics where relativistic heavy nuclei bombard a light-mass target. The neutron beam generated at $0^{\circ}$ has a Gaussian shape with a maximum at the energy of the projectile nucleons and an energy resolution $\sigma_{\mathrm{E}} / E<4 \%$ above $6 \mathrm{GeV}$.
\end{abstract}

Keywords: high-energy neutron beam; heavy ion fragmentation; neutron emission.

PACS numbers: 25.40.Sc, 29.25.Dz.

\section{Introduction}

Experiments with beams of monoenergetic high-energy neutrons are a dream of physicists. Such neutrons cannot be produced with available accelerators, but we can generate quasi-monoenergetic neutrons in special nuclear reactions induced by accelerated protons and heavy ions.

Neutrons with energy from tens to hundreds of $\mathrm{MeV}$ are usually produced by means of the ${ }^{7} \mathrm{Li}(p, n)$ reaction, and this method is applied in many laboratories. ${ }^{1}$ Neutron beams with higher energies up to several $\mathrm{GeV}$ were generated by a stripping process with a deuteron beam at Saclay ${ }^{2,3}$ and JINR. ${ }^{4}$

The relativistic beams of heavy $\mathrm{Pb}$ nuclei at SPS/CERN and $\mathrm{Au}$ at AGS/BNL give a new opportunity to produce a beam of quasi-monoenergetic neutrons with the highest energy, i.e. above $10 \mathrm{GeV}$, by fragmentation of heavy ions in collisions with targets of light-mass nuclei. This is a very promising feature to study neutron-proton and neutronnucleus collisions at SPS energies.

In this paper, characteristics of these ultra-relativistic neutrons are studied with the purpose of estimating the possibility of neutron beam production with available heavy ion

This is an Open Access article published by World Scientific Publishing Company. It is distributed under the terms of the Creative Commons Attribution 3.0 (CC-BY) License. Further distribution of this work is permitted, provided the original work is properly cited. 
beams at SPS and AGS. This goal is achieved by: (i) the analysis of the neutron production double-differential cross sections for $p+\mathrm{Pb}$ and $\mathrm{C}+\mathrm{Pb}$ interactions above 0.5 $\mathrm{GeV}$ with a Moving Source Model (MSM), (ii) the Lorentz transformation of the obtained neutron distributions to the inverse kinematics, and (iii) the estimation of the neutron beam characteristics. Thus, the base of this work is a set of available experimental data on the neutron production in high-energy reactions and MSM analysis of these data. ${ }^{5-8}$

\section{Neutron Production in High Energy Reactions}

During the last quarter of the previous century, many experiments were carried out with beams of protons and light-mass heavy ions for the study of nuclear matter characteristics at very high excitation. Collisions of light projectiles with heavy nuclei are an effective method to produce hot nuclei with small excitation of collective modes. The data on particle emission characteristics is the main information coming from the measurements. These data are important both for understanding the reaction mechanisms and for verification and development of theoretical models and codes.

Nucleon-nucleon inelastic cross sections dramatically increase above $500 \mathrm{MeV}$ due to the appearance of new channels - the excitation of baryon resonances. In this energy range, this mechanism plays the main role in the energy transfer from a beam ion to a target nucleus. The free path of a high energy projectile in nuclear matter becomes $\sim 1 \mathrm{fm}$, and in central collisions, a heavy nucleus looks like a very thick target, which effectively absorbs the beam ion energy by internuclear cascade. This is the first stage of the reaction with a duration of $\sim 10 \mathrm{fm} / c$ and emission of some energetic nucleons and mesons.

The result of the cascade in a central collision is formation of a hot source, i.e. fireball, consisting of nucleons, baryon resonances, pions, and other mesons. After thermalization and expansion, the fireball decays with emission of nucleons, low energy pions, and light charged particles. The lifetime of this second stage is $\sim 20-30 \mathrm{fm} / c$.

After the second stage, a large part of energy is transferred to the residual nuclear system with excitation energy up to several $\mathrm{MeV}$ per nucleon. Such a nuclear system is unstable to decay. The thermalization and expansion of the nuclear remnant lead to the decay with multiple nucleon and fragment emission. The fragmentation occurs at a freeze-out point. The third stage of the nuclear system decay takes $\sim 80-100 \mathrm{fm} / c$. The fourth source of neutrons is de-excitation of heavy fragments by means of neutron evaporation. These excited secondary nuclei are produced both in peripheral and central collisions.

The analysis of experimental data on charged particle, fragment, and neutron production in high-energy reactions supports this picture of the nuclear system evolution and decay with four main stages-sources shown schematically in Fig. 1. 


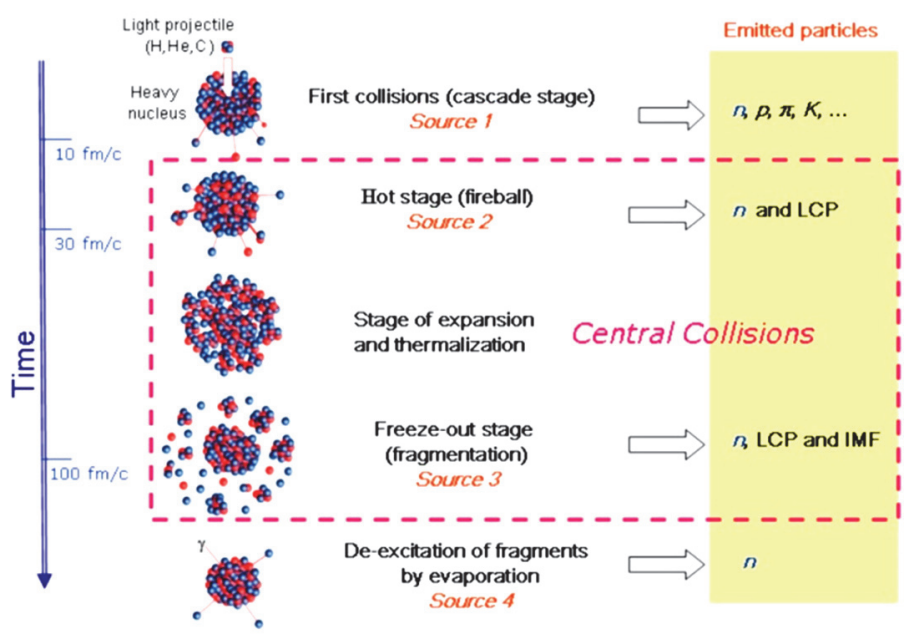

Fig. 1. A picture of the nuclear system evolution and decay with four main stages-sources of particle emission.

The source contributions form a shape of the energy spectra of neutrons at large angles to the beam axis. This approach is realized in a Moving Source Model (MSM) with four neutron sources: (1) $S_{1}$, cascade stage; (2) $S_{2}$, fireball decay; (3) $S_{3}$, multifragmentation, and (4) $S_{4}$, evaporation. The second and third sources occur only in central collisions. The MSM expression is based on an assumption about thermal equilibrium in each particle source and isotropic emission in the source frame. The formula has four terms in correspondence with the number of neutron sources:

$$
\frac{d^{2} \sigma}{d E_{k i n} d \Omega}=\sum_{i=1}^{4} p A_{i} \exp \left\{-\left(\frac{E_{k i n}+m-p \beta_{i} \cos \theta}{\left(1-\beta_{i}^{2}\right)^{1 / 2}}-m\right) / T_{i}\right\},
$$

where $E_{\text {kin }}$ and $\theta$ are the kinetic energy $(\mathrm{MeV})$ and the angle of the emitted neutron in the lab frame, respectively, $p$ is the neutron momentum, $\Omega$ is the solid angle, and $T$ is the temperature parameter $(\mathrm{MeV})$. There are three parameters for each source: the amplitude $A_{\mathrm{i}}$, the temperature $T_{\mathrm{i}}$ and the normalized velocity $\beta_{\mathrm{I}}=V_{\mathrm{i}} / c$. It is expected that the temperature and velocity parameters of the different sources have the following relations: $T_{1}>T_{2}>T_{3}>T_{4}$ and $\beta_{1}>\beta_{2}>\beta_{3}>\beta_{4}$.

Production of high energy neutrons at small angles to the beam axis in elastic and quasi-elastic interactions and projectile fragmentation is not taken into account in our consideration. This is because in inverse kinematics, where relativistic heavy nuclei collide with light mass nuclei of a target, these neutrons give some small contribution to the low energy region, and it is out of our interest.

The MSM analysis of the double-differential cross sections for neutron production in collisions of relativistic $p,{ }^{4} \mathrm{He}$, and $\mathrm{C}$ with $\mathrm{Pb}$ nuclei is described in detail in Refs. 5-8. It was shown that the sources $\mathrm{S}_{2}$ and $\mathrm{S}_{3}$ give about $40 \%$ of all neutrons in the reactions. The mean number of neutrons increases with beam energy with a maximum of 35 neutrons 
reached at higher energies. An example of the fitting of neutron energy spectra measured at different angles for interactions of $2 \mathrm{GeV}$ protons with lead nuclei is shown in Fig. 2.

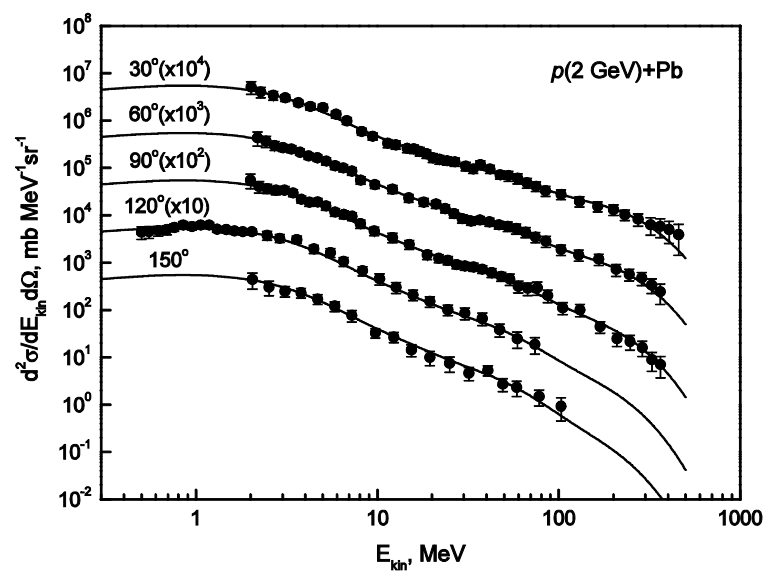

Fig. 2. The neutron production double-differential cross sections for $p+\mathrm{Pb}$ collisions at an energy of $2 \mathrm{GeV}$ : points are experimental, ${ }^{5,7}$ curves are MSM fit.

Contributions of the sources to the energy spectra of neutrons are shown in Fig. 3 for $p+\mathrm{Pb}$ and $\mathrm{C}+\mathrm{Pb}$ reactions. At an energy of several $\mathrm{GeV}$, the source temperatures are $T_{1}$ $\approx 70 \mathrm{MeV}, T_{2} \approx 21 \mathrm{MeV}, T_{3} \approx 4.7 \mathrm{MeV}$, and $T_{4} \approx 1.6 \mathrm{MeV}$, and the source velocities were estimated as $\beta_{1} \approx 0.2, \beta_{2} \approx 0.03, \beta_{3} \approx 0.005$, and $\beta_{4} \approx 0$.

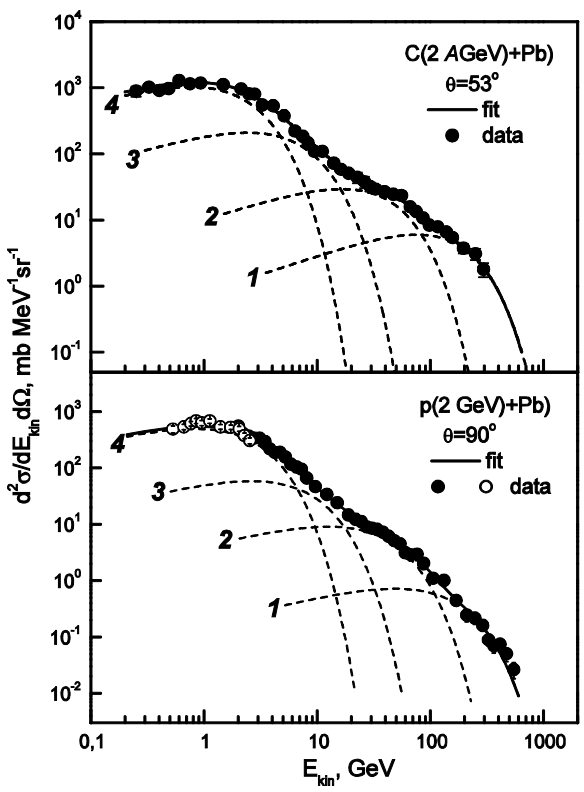

Fig. 3. The source temperatures as a function of the beam energy. 
The contributions of different sources to the mean multiplicity of neutrons in $p+\mathrm{Pb}$ and $\mathrm{C}+\mathrm{Pb}$ collisions are shown in Fig. 4. For both reactions, the contributions increase with the source number, where minimum values are given by the cascade stage and maximum contribution comes from the evaporation stage. The ratio between the source contributions may probably change somewhat at higher beam energies. To clarify this question, new experiments on neutron production above $10 \mathrm{GeV}$ are required.

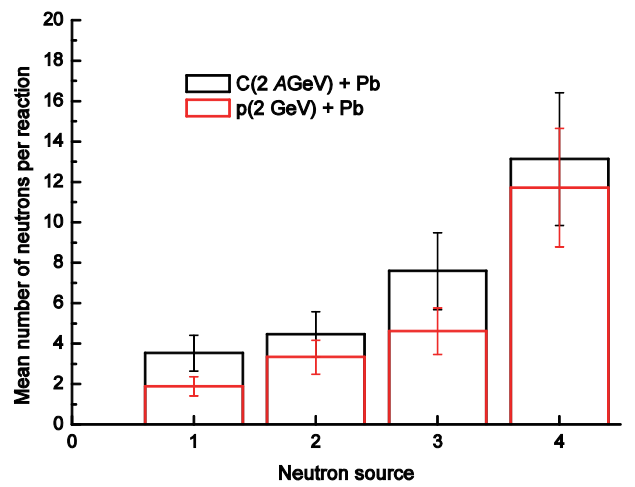

Fig. 4. Contributions of different sources to the mean multiplicity of neutrons in $p+\mathrm{Pb}$ and $\mathrm{C}+\mathrm{Pb}$ collisions.

\section{Neutrons from Fragmentation of Relativistic Heavy Nuclei}

Our goal is to assess the characteristics of high-energy neutrons produced by fragmentation of relativistic $\mathrm{Pb}$ ions in interactions with light mass nuclei. This is achieved by the Lorentz transformation of neutron distributions obtained by the MSM fitting of experimental data for high energy reactions induced by proton and carbon beams as discussed above. In the inverse kinematics, the neutron distributions dramatically change as shown in Fig. 5 for $\mathrm{Pb}+\mathrm{C}$ and $\mathrm{Pb}+p$ collisions.
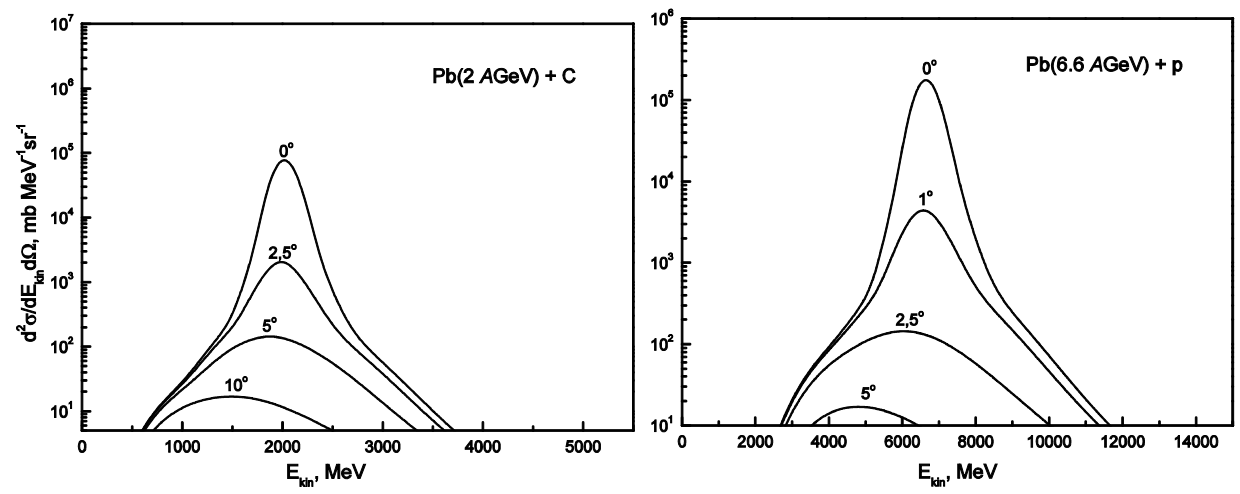

Fig. 5. The energy spectra of neutrons produced at different angles $\theta$ in $\mathrm{Pb}+\mathrm{C}$ and $\mathrm{Pb}+p$ collisions at energies of $2 \mathrm{GeV} / \mathrm{u}$ and $6.6 \mathrm{GeV}$, respectively. 
The maximum intensity is observed at $\theta=0^{\circ}$ along the beam axis, and the neutron intensity rapidly drops down with an increase of the angle. For $\mathrm{Pb}+p$ collisions at $1 \mathrm{GeV} / \mathrm{u}$, approximately $34 \%$ of all neutrons move into a cone with $\theta<1^{\circ}$, while at an energy of $6.6 \mathrm{GeV} / \mathrm{u}$, already $73 \%$ of all neutrons move into the same cone.

The neutron distributions slightly depend on the type of target nuclei, proton and carbon, at energies of several $\mathrm{GeV} / \mathrm{u}$. The angular distribution becomes narrower in the forward direction with the beam energy as is clearly seen in Fig. 6 where the results for $\mathrm{C}$ $+\mathrm{Pb}$ collisions at $2 \mathrm{GeV} / \mathrm{u}$ and $p+\mathrm{Pb}$ collisions at $6.6 \mathrm{GeV}$ are shown in linear scale. At small angles, the neutron spectra have a peak shape, and the position of the maximum is equal to the kinetic energy per nucleon of the ion beam. The peak at $0^{\circ}$ is well-fitted with a Gaussian distribution, as shown in Fig. 7. The relative energy spread decreases with the beam energy and energy of neutrons. This dependence is shown in Fig. 8. It is clearly seen that the beam becomes similar to monoenergetic with a small energy of dispersion $\sigma_{\mathrm{E}} / E \leq 4 \%$ above $6 \mathrm{GeV}$.
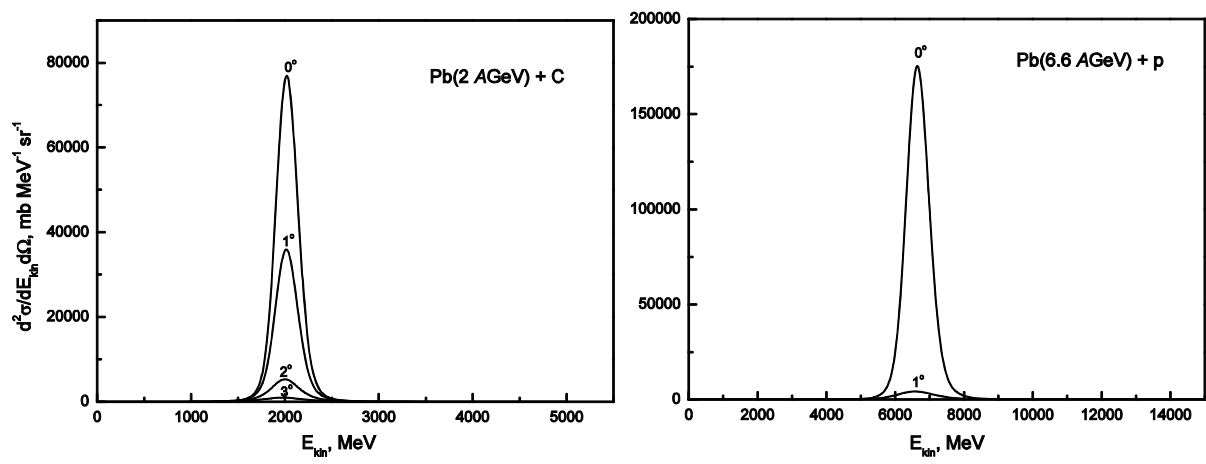

Fig. 6. The energy spectra of neutrons at small angles for $\mathrm{Pb}+\mathrm{C}$ and $\mathrm{Pb}+p$ collisions at energies of 2 and 6.6 $\mathrm{GeV} / \mathrm{u}$, respectively.
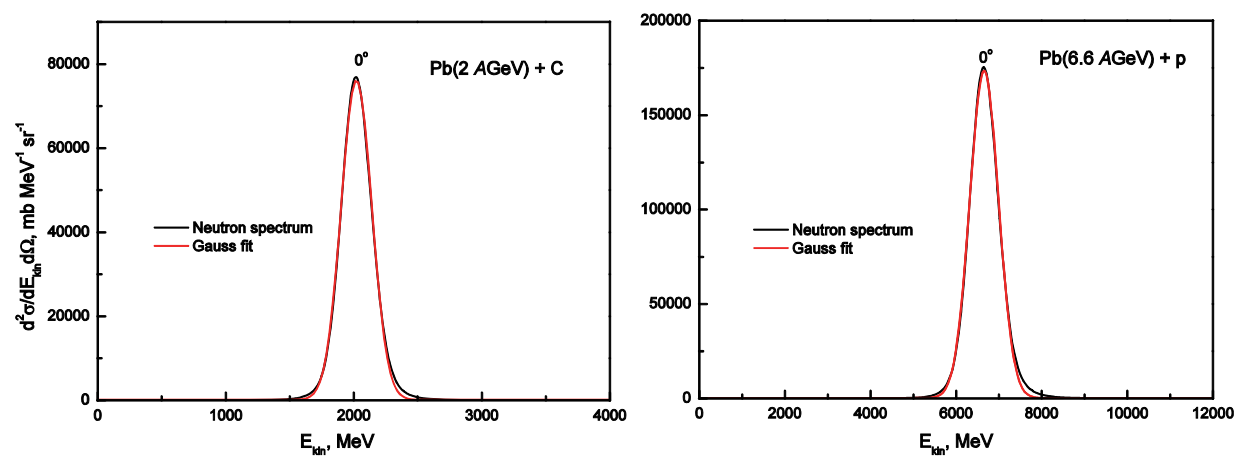

Fig. 7. Neutron spectra at $0^{\circ}$ with Gaussian fits for $\mathrm{Pb}+\mathrm{C}$ and $\mathrm{Pb}+p$ collisions. 


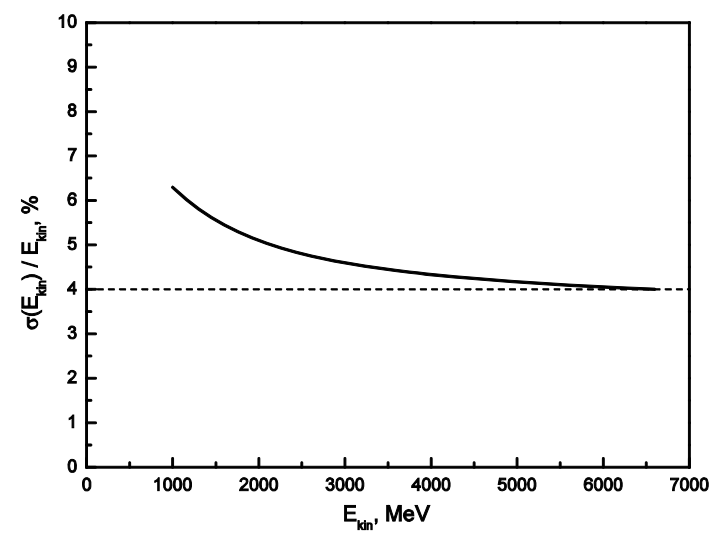

Fig. 8. The energy dispersion as a function of neutron energy.

\section{Beam of High Energy Neutrons}

Unlike a beam of charged particles, a neutron beam cannot be focused with a magnet system. Therefore, the transverse size of the beam increases with distance. It depends on the beam energy and can be decreased by collimation with a simultaneously decreasing neutron intensity. The radius of the beam spot corresponding to $75 \%$ of all neutrons was calculated for the beam line length of $10 \mathrm{~m}$. The result is shown in Fig. 9 as a function of heavy ion energy with extrapolation to AGS and SPS energies. At the maximum energy of AGS, the spot radius is $11 \mathrm{~cm}$, and for the maximum energy of SPS, it becomes 0.86 $\mathrm{cm}$ or $8.6 \mathrm{~cm}$ at a distance of $100 \mathrm{~m}$. A simple estimation shows that for a $4 \%$ interaction target, the neutron beam intensity is similar to the intensity of the $\mathrm{Pb}$ ion beam of SPS.

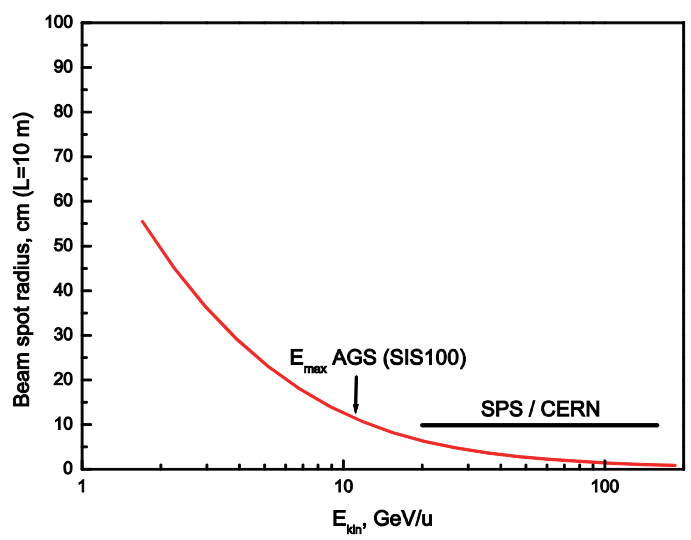

Fig. 9. The transverse size of the neutron beam corresponding to $75 \%$ of all emitted neutrons at a distance of $10 \mathrm{~m}$ as a function of heavy ion energy. 
A schematic of the neutron beam line is shown in Fig. 10, which consists of a target, a beam-bending magnet behind the target, and a system of collimators. Any light mass medium such as polyethylene may be used as the neutron production target. At SPS energies with the ion beam intensity up to $10^{6} \mathrm{ion} / \mathrm{s}$, neutron pulses produced in interactions of lead ions in a target can be selected with two Cherenkov gas beam counters (BC1 and $\mathrm{BC} 2$ in Fig. 10) placed in front and behind the target and used in anticoincidence. Also, the first counter based on MCP-PMT or SiPM with picosecond time resolution might be used as a start detector in the TOF measurements.

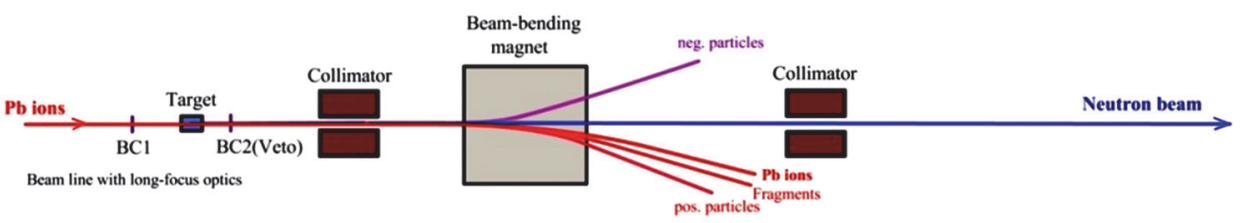

Fig. 10. A schematic of the neutron beam channel.

The beam characteristics are:

- The beam energy depends on the accelerator beam energy per nucleon.

- The energy resolution becomes $\sigma_{\mathrm{E}} / E_{\mathrm{kin}} \leq 4 \%$ at $E_{\mathrm{kin}} \geq 6 \mathrm{GeV}$.

- The beam intensity depends on the accelerator beam intensity and energy, thickness and type of the target, and collimation.

- The beam structure is defined by the accelerator beam structure, and a picosecond microstructure comes from the multiple neutron production in heavy ion interaction in the target, with a number of neutrons from several to hundred emitted simultaneously,

- The beam transverse size depends on the ion beam energy, beam optics, and collimation.

The estimations performed show that the $\mathrm{Pb}$ beam at SPS/CERN is the most convenient for generation of a high-energy neutron beam with small energy dispersion.

\section{Parasitic Neutron Beams}

Another important topic is the generation of parasitic neutron beams in interactions of high-energy heavy ions with matter in a beam channel. The possible sources are the beam detectors, windows of beam pipe, and air. For example, even a $100-\mu \mathrm{m}$ diamond start detector can give essential forward background of high energy neutrons.

The next source is nuclear interactions in stripping foils of accelerators. At CERN, an aluminum stripping foil with a thickness of 0.5 (or 0.8 ) $\mathrm{mm}$ is used for production of $\mathrm{Pb}^{+82}$ ions at an energy of $5.9 \mathrm{GeV} / \mathrm{u}$. The $\mathrm{Pb}$ ion interactions in the stripping foil produce 
a parasitic beam of $5.9 \mathrm{GeV}$ neutrons at $0^{\circ}$. This effect must be taken into account for radiation shielding design around stripping foils and target stations.

\section{Conclusion}

Picosecond pulses of $\mathrm{GeV}$ neutrons at $0^{\circ}$ with an energy resolution of $\sigma_{\mathrm{E}} / E_{\mathrm{kin}} \leq 4 \%$ can be realized with available beams of $\mathrm{Pb}$ and $\mathrm{Au}$ ions at SPS/CERN and AGS/BNL. The interaction rate in the neutron production target defines the rate of neutron pulses. The parasitic beams of high energy neutrons induced by heavy ions in the beam line materials have to be taken into account in projects of new experimental setups, beam lines, and radiation shielding. The experimental neutron data base for the high energy region is rather poor, and new experiments for the study of neutron production at energies above $10 \mathrm{GeV}$ are required.

\section{References}

1. S. Pom, D. T. Bartlett, S. Mayer, G. Reitz, S. Röttger, M. Silari, F. D. Smit, H. Vincke and H. Yasuda, High-energy quasi-monoenergetic neutron fields: existing facilities and future needs, EURADOS Report 2013-02, 2013.

2. G. Bizard, F. Bonthonneau, J. L. Laville, F. Lefebvres, J. C. Malherbe, R. Regimbart, J. Duflo and F. Plouin, Nucl. Instr. Meth. 111, 445 (1973).

3. J. F. Lecolley, C. Varignon, J. Thun, F. Borne, E. Martinez et al., Eur. Phys. J. A. 5, 321 (1999).

4. A. Kirillov, L. Komolov, A. Kovalenko, E. Matyushevsky, A. Nomofilov, P. Rukoyatkin, V. Sharov, A. Starikov, L. Strunov and A. Svetov, Relativistic polarized neutrons at the laboratory of high energy physics, JINR, JINR Preprint E13-96-210 (Dubna, 1996).

5. V. I. Yurevich, R. M. Yakovlev and V. G. Lyapin, Physics of Atomic Nuclei 69, 1469 (2006).

6. V. I. Yurevich, Physics of Particles and Nuclei 40, 49 (2009).

7. V. I. Yurevich, R. M. Yakovlev, V. G. Lyapin, Physics of Atomic Nuclei 74, 253 (2011).

8. V. I. Yurevich, R. M. Yakovlev, V. G. Lyapin, Physics of Atomic Nuclei 75, 192 (2012). 